\title{
PRIMEIRO CONSENSO BRASILEIRO PARA TROMBÓLISE NO ACIDENTE VASCULAR CEREBRAL ISQUÊMICO AGUDO
}

\author{
Sociedade Brasileira de Doenças Cerebrovasculares (SBDCV) ${ }^{1}$
}

\begin{abstract}
RESUMO - Este texto apresenta a síntese das conclusões do I Consenso Brasileiro para Trombólise no Acidente Vascular Cerebral. Tratou-se de reunião promovida e coordenada pela Sociedade Brasileira de Doenças Cerebrovasculares, com neurologistas especializados em doenças cerebrovasculares, que analisaram e discutiram os requisitos assistenciais e profissionais, bem como as diretrizes e os protocolos clínicos, para o uso de trombólise em pacientes com acidente vascular cerebral isquêmico agudo.
\end{abstract}

PALAVRAS-CHAVE: acidente vascular cerebral agudo, trombólise endovenosa, consenso brasileiro.

\begin{abstract}
Brazilian consensus for the thrombolysis in acute ischemic stroke
ABSTRACT - This consensus is the result of a recent meeting to establish the ideal approach for thrombolysis in acute stroke patients in Brazil. Some peculiarities concerning the emergency rooms, stroke units, available equipments and stroke teams are considered in order to characterize the stroke centers. Protocols concerning the use of thrombolytic drugs are reviewed. This is the official guideline for thrombolysis in acute stroke of the Brazilian Society of Cerebrovascular Disease.
\end{abstract}

KEY WORDS: stroke, cerebrovascular disease, thrombolysis, Brazilian consensus.

As doenças cerebrovasculares têm grande impacto sobre a saúde da população, situando-se, conforme o ano e o Estado da Federação, entre a primeira e terceira principal causa de mortalidade no Brasil ${ }^{1}$. Estas doenças são compostas por grupo heterogêneo de transtornos vasculares de diferentes etiologias $^{2}$. Estima-se que cerca de $85 \%$ dos acidentes vasculares encefálicos sejam de origem isquêmica e $15 \%$ hemorrágicos. Dentre os hemorrágicos, cerca de $10 \%$ são hemorragias intraparenquimatosas e $5 \%$ hemorragias subaracnóideas ${ }^{3,4}$. A aprovação pela "Federal Drug Administration", nos Estados Unidos da América, em Junho de 1996, do ativador do plasminogênio tissular recombinante (rt-PA) como trombolítico para o uso em casos selecionados deacidente vascular cerebral (AVC) isquêmico agudo, veio reforçar a estratégia estabelecida nos últimos anos de considerar o AVC como uma emergência médica ${ }^{5-7}$. Existe uma "janela terapêutica" para o tratamento do AVC, ou seja, um momento ótimo para intervir nos processos patológicos desencadeados pela isquemia cerebral no sentido de minimizar o dano ao sistema nervoso central ${ }^{8}$. Esta janela terapêutica, na maioria das vezes, tem uma duração de poucas horas, o que determina a necessidade de rapidez no atendimento às pessoas que apresentam um AVC agudo ${ }^{9-13}$. Várias entidades, como o "European Stroke Council", a "International Stroke Society", a "American Academy of Neurology", a "American Stroke Association", a Sociedade Brasileira de Doenças Cerebrovasculares (SBDCV) e a Organização Mundial da Saúde, têm divulgado orientações sobre o manejo das doenças cerebrovasculares ${ }^{12,14-21}$. Todas chamam a atenção para a necessidade de mudança de atitudes com relação a estas doenças, tanto da população como dos profissionais e instituições de saúde, no sentido de considerar a fase aguda como uma situação ameaçadora à vida.

Para que isto ocorra, torna-se necessária a educação das pessoas para reconhecerem quais sinto-

\footnotetext{
'Sociedade Brasileira de Doenças Cerebrovasculares, São Paulo SP, Brasil: Redatores - Cesar Noronha Raffin, Rubens José Gagliardi, Ayrton Roberto Massaro, Jefferson Gomes Fernandes, Aroldo Luiz Bacellar, Soraia Ramos Cabette Fábio.Participantes - Alexandre L. Longo, Aroldo Luiz Bacellar, Ayrton Roberto Massaro, Carla Heloísa Cabral Moro, Cesar Noronha Raffin, Charles André, Edison Matos Nóvak, Elza Dias Tosta, Fábio luji Yamamoto, Gabriel Rodríguez De Freitas, Jamary Oliveira Filho, Jefferson Gomes Fernandes, Jorge ElKadum Noujaim, José Ibiapina Siqueira Neto, Ibsen Thadeo Damiani, Marcelo Gabriel Veja, Marcia Maiumi Fukujima, Roberto de Magalhães Carneiro de Oliveira, Rubens José Gagliardi, Sérgio Roberto Haussen, Soraia Ramos Cabette Fábio, Viviane Flumignan Zétola.
}

Recebido 18 Março 2002, recebido na forma final 13 Maio 2002. Aceito 18 Maio 2002.

Sociedade Brasileira de Doenças Cerebrovasculares - A/C Dr. Cesar Noronha Raffin, Presidente - Avenida Angélica 916/305 - 01223-000 São Paulo SP - Brasil. 
mas possam ser indicativos de um AVC e que estes sintomas devem determinar a busca de um atendimento emergencial ${ }^{12,21}$. Há a necessidade, também, do treinamento dos profissionais de saúde, especialmente que trabalham em serviços de ambulância e de urgência, para o diagnóstico e manejo inicial adequado a estes doentes ${ }^{7}$. Da mesma forma, as instituições de saúde que prestam assistência médica precisam adequar a sua estrutura para o atendimento emergencial do paciente com AVC, disponibilizando, entre outros aspectos, um acesso rápido à tomografia computadorizada de crânio eatendimento neurológico a estes doentes ${ }^{12,21}$. Com o propósito do uso de trombolítico para o AVC agudo, torna-se importante a participação de especialistas com experiência no diagnóstico das doenças cerebrovasculares e na interpretação de exames de neuroimagem $^{5,7}$.

Com este objetivo, a Sociedade Brasileira de Doenças Cerebrovasculares (SBDCV), sendo o grupo de trabalho em patologia vascular da Academia Brasileira de Neurologia, estabeleceu os protocolos e recomendações para a orientação e a qualificação para o uso de trombolítico nos pacientes com AVC isquêmico agudo no Brasil.

\section{A. RECOMENDAÇÕES PARA CRIAÇÃO DOS CENTROS DE REFERÊNCIA PARA TROMBÓLISE EM ACIDENTE VASCULAR CEREBRAL ISQUÊMICO}

1. A SBDCV, representando a Academia Brasileira de Neurologia, é a responsável pela avaliação e certificação dos Centros de Referência para trombólise em pacientes portadores de doenças cerebrovasculares.

1.1. Será constituída uma comissão nacional de membros da SBDCV com a finalidade específica de avaliação e certificação periódica destes centros.

2. Os Centros de Referência serão classificados em 3 níveis (nível $A, B$ e C) de acordo com os critérios discriminados abaixo.

2.1. Nível C: o centro que apresentar todas as seguintes características:

2.1.1. Equipe organizada com protocolos clínicos e assistenciais escritos para atendimento dos pacientes com AVC, coordenada por neurologista clínico. Fazem parte ainda da equipe a enfermagem especializada em emergência e atendimento de pacientes com AVC e a equipe de reabilitação neurológica.

2.1.2. O paciente deve ser atendido em Serviço de Emergência com capacidade para monitoração contínua sob o ponto de vista cardiovascular e respiratório.

2.1.3. Deve dispor obrigatoriamente de Unidade de Terapia Intensiva (UTT); Laboratório 24 horas e Tomografia Computadorizada (TC) disponível 24 horas.
2.1.4. Disponibilidade neurocirúrgica 24 horas.

2.1.5. Disponibilidade de Banco de Sangue.

2.2. Nível B: o centro que apresentar, além do descrito para o nível $C$, todos os seguintes recursos:

2.2.1. Ressonância magnética.

2.2.2. Ultra-som vascular intra e extracraniano.

2.2.3. Ecocardiografia transtorácica e transesofágica.

2.3. Nível A: o centro que apresentar, além do descrito para o Nível B, todos os seguintes recursos:

2.3.1. Ressonância magnética com técnicas para angiografia, difusão e perfusão.

2.3.2. Angiografia digital.

2.3.3. Neurorradiologia intervencionista.

2.3.4. Unidade de AVC agudo com área física especifica.

\section{B. CRITÉRIOS PARA FIRMAR O DIAGNÓSTICO E AVALIAÇÃO CLÍNICA}

1. É obrigatória a imediata participação do neurologista o mais rápido possível sempre que houver suspeita de AVC.

2. Os critérios clínicos para o diagnóstico do AVC consideram:

2.1. Déficit focal ao exame neurológico, com ou sem distúrbio de consciência.

2.2. Início súbito, agudo ou rapidamente progressivo.

2.3. Sinais clínicos persistentes até o início da trombólise.

Observações:

a. Os seguintes sinais e sintomas quando presentes isoladamente não são necessariamente suficientes para o diagnóstico de AVC: alteração sensitiva monossegmentar, disartria, disfagia, diplopia e vertigem.

b. Merecem especial atenção os doentes que se apresentam com comprometimento do nível de consciência, flutuante ou não; nestes casos deve-se procurar minuciosamente a possibilidade de sinais adicionais de lesão no território arterial vertebrobasilar.

3. Confirmação diagnóstica: A confirmação diagnóstica deverá ser feita através de neuroimagem ${ }^{22}$. A análise do exame deverá ser feita por médico experiente em neuroimagem, que deverá reconhecer os critérios de exclusão para o uso de trombolítico ${ }^{7,13,21,23 .}$.

3.1. Tomografia computadorizada: É obrigatória a realização da tomografia de crânio sem contraste. Recomenda-se que seja realizada em até 30 minutos, contados desde a admissão do paciente: é ideal a leitura do exame até 45 minutos. A utilização do contraste dependerá do quadro clínico e do resultado do exame simples.

3.2. Ressonância magnética: Quando disponível e realizável em tempo hábil, pode-se utilizar a ressonância magnética do crânio, de preferência com as técnicas de difusão e perfusão ${ }^{24-27}$. É importante que a realização deste examenão aumenteo tempo de espera para o uso do trombolítico por causa da janela terapêutica de três horas. 
Observação: Deve-se considerar que a sensibilidade da ressonância para diagnóstico da hemorragia subaracnóidea é menor do que a da tomografia computadorizada.

4. Diagnóstico diferencial: É essencial a exclusão das seguintes condições clínicas: epilepsia eestados pós-comiciais, tumores, trauma crânio-encefálico, hemorragias intracranianas (espontâneas ou traumáticas), enxaqueca, amnésia global transitória, distúrbios metabólicos (principalmente hipo e hiperglicemia), infecções do sistema nervoso central, esclerose múltipla, labirintopatias, efeitos de medicamentos ou drogas de abuso e distúrbios psicossomáticos ${ }^{21}$.

5. Avaliação clínica: A avaliação clínica neurológica deve ser feita por neurologista habilitado, e procurar fundamentalmente confirmar a suspeita clínica, conferir os critérios de inclusão e exclusão para o uso de trombolítico e obter parâmetros para acompanhamento evolutivo do paciente ${ }^{7,12}$. A avaliação inicial deve ser breve e sistemática, priorizando:

5.1. Tempo comprovado de início dos sintomas.

5.2. Avaliar critérios de exclusão.

5.3. Exame clínico com ênfase para sinais vitais - pressão arterial (PA), frequência cardíaca, frequência respiratória e temperatura.

5.4. Exame neurológico.

6. Suporte clínico e acompanhamento: Na avaliação clínica inicial e sequencial, é obrigatório o uso de método de monitoração do nível de consciência e do déficit neurológico. Recomenda-se para este fim a aplicação da escala de Glasgow ${ }^{28}$ e a escala do AVC do National Institutes of Health (NIHSS) ${ }^{29,30}$, respectivamente. Durante a infusão do rt-PA e nas 24 horas seguintes, o doente deve permanecer de preferência em Unidade de AVC ou Unidade de Tratamento Intensivo com disponibilidade de serviço de neurocirurgia, hematologia, banco de sangue eneuroimagem, para monitorização da PA (medir a cada 15 minutos nas primeiras 2 horas, a cada 30 minutos nas próximas 6 horas e a cada 60 minutos até completar 24 horas), oximetria de pulso, monitoração cardíaca externa e, especialmente, para a deteç̧ão precoce de sinais e sintomas que indiquem piora neurológica e que obriguem à tomada de medidas específicas 7,12 . Recomenda-se a utilização de método para a avaliação da eficácia da trombólise, por exemplo, Doppler transcraniano, angiotomografia ou angiorressonância ${ }^{7}$. Sugere-se avaliação periódica durante a internação do doente e após a alta hospitalar com escalas específicas, por exemplo, NIHSS, Barthel e Rankin 29-31 .

\section{RECOMENDAÇÕES AO ATENDIMENTO}

1. Fase pré-hospitalar

1.1. Reconhecimento dos sinais e sintomas. Recomenda-se o estabelecimento de estratégias de educação pública para o reconhecimento precoce dos sinais e sinto- mas do AVC e para a rápida busca de assistência médica (senviços de emergência móvel ou emergência hospitalar). 1.2. Ativação do serviço de emergência móvel.Os serviços de emergência médica devem dar prioridade máxima ao atendimento ao paciente com AVC agudo; reconhecer e manejar o AVC através de protocolos específicos, considerando a possibilidade do uso de trombolítico; e transportar rapidamente o paciente a um Centro de Referência de AVC, de preferência contactando-o previamente. Sugere-se que estes protocolos específicos incluam, entre outras orientações, uma escala de AVC pré-hospitalar, o registro do horário do início dos sintomas e a orientação de não reduzir, a princípio, a pressão arterial7,12,21.

\section{Fase hospitalar}

2.1. Avaliação e suporte vital: Deve envolver o " $A B C$ " ("Airway, Breathing, Circulation") dos cuidados ao paciente crítico e avaliação médica geral ${ }^{13}$.

2.2. Avaliação neurológica emergencia|l ${ }^{13,31}$ : Esta avaliação deve contemplar os seguintes itens:

2.2.1. Diagnóstico diferencial.

2.2.2. Nível de consciência (Escala de Coma de Glasgow)

2.2.3. Tipo de AVC (hemorragia $x$ isquemia)

2.2.4. Localização (sistema carotídeo $x$ vertebrobasilar)

2.2.5. Gravidade (NIHSS ${ }^{29,30}$ )

2.3. Investigação diagnóstica emergencial, dirigida ao uso do trombolítico 31 :

2.3.1. Laboratorial: hemograma e plaquetas, sódio, potássio, creatinina, glicemia, tempo de tromboplastina parcial ativada (TTPA), tempo de protrombina (TP) e tipagem sanguínea.

2.3.2. Eletrocardiograma (ECG).

2.3.3. Radiografias do tórax.

2.3.4. Tomografia computadorizada de crânio (sem contraste).

2.4. Recomendações para metas de tempos de atendimento: Os tempos apresentados na Tabela 1 representam metas razoáveis a serem atingidas pelos Centros de Refe-

Tabela 1. Recomendações do National Institute of Neurological Disorders and Stroke (NINDS) para candidatos potenciais ao trombolítico.

\begin{tabular}{lc}
\hline & $\begin{array}{c}\text { Tempo } \\
\text { recomendável }\end{array}$ \\
\hline Da admissão à avaliação médica & 10 minutos \\
Da admissão ao TC de crânio (término) & 25 minutos \\
Da admissão ao TC de crânio (interpretação) & 45 minutos \\
Da admissão à infusão do rt-PA & 60 minutos \\
Disponibilidade do neurologista & 15 minutos \\
Disponibilidade do neurocirurgião & 2 horas \\
Da admissão ao leito monitorizado & 3 horas \\
\hline
\end{tabular}


rência de AVC, para permitir a inclusão do maior número possível de pacientes para o tratamento com o trombolítico 5,6 .

2.5. A escolha do trombolítico: A estreptoquinasefoi avaliada em diversos estudos, sendo o seu uso endovenoso (EV) proscrito por causa dos altos índices de hemorragia e mortalidade por hemorragia demonstrada nestes estudos (nível de evidência I) ${ }^{32-35}$. O uso do ativador do plasminogênio tissular (rt-PA), quando administrado por via endovenosa ao paciente nas primeiras 3 horas, demonstrou importante diminuição na incapacidade funcional no grupo que utilizou a droga em relação ao placebo, sendo, portanto o único medicamento recomendado para o tratamento na fase aguda do AVC isquêmico (nível de evidência I)5.

3. Protocolo para uso do rt-PA: Para maior segurança, sua aplicação deve respeitar rigidamente os critérios a seguir 5 :

3.1. Critérios de inclusão:a) AVC isquêmico em qualquer território encefálico; b) Possibilidade de se estabelecer precisamente o horário do início dos sintomas; c) Possibilidade de se iniciar a infusão do rt-PA dentro de 3 horas após o início dos sintomas (caso os sintomas sejam observados ao acordar, deve-se considerar o último horário no qual o paciente foi observado em condições normais); d) Realização de tomografia computadorizada do crânio sem evidência de hemorragia; e) Idade superior a 18 anos.

3.2. Critérios de exclusão: a) Uso de anticoagulantesorais com tempo de pró-trombina (TP) $>15$ segundos ( $\mathrm{RNI}>1,7$ ); b) Uso de heparina nas últimas 48 horas com TTPa elevado; c) AVC isquêmico ou traumatismo crânio-encefálico grave nos últimos 3 meses; d) História pregressa de alguma forma de hemorragia cerebral (hemorragia subaracnóidea ou cerebral) ou história de malformação vascular cerebral ou aneurisma cerebral; e) TC de crânio com sinais precoces de infarto (apagamento de sulcos cerebrais, hipodensidade ou edema) em mais de um terço do território da artéria cerebral media; f) PA sistólica $>185 \mathrm{mmHg}$ ou PA diastólica $>110 \mathrm{mmHg}$ (em 3 ocasiões, com $10 \mathrm{mi}$ nutos de intervalo) ou que necessite de tratamento antihipertensivo EV contínuo; g) Sintomas neurológicos melhorando rapidamente; $h$ ) Déficits neurológicos leves (NIHSS $<4$ ), exceto por afasia isolada ; h) Cirurgia de grande porte ou procedimento invasivo dentro das últimas 2 semanas; i) Hemorragia geniturinária ou gastrointestinal (nas últimas 3 semanas), ou história de varizes esofagianas ou doença inflamatória intestinal; j) Punção arterial não compressível ou biópsia na última semana; k) Coagulopatia com TP prolongado ( $>15$ segundos), TTPa elevado, ou plaquetas < $100000 / \mathrm{mm}^{3}$; l) Glicemia < $50 \mathrm{mg} / \mathrm{dl}$ ou $>400$ $\mathrm{mg} / \mathrm{dl} ; \mathrm{m}$ ) Crise convulsiva precedendo ou durante a instalação do AVC; n) Evidência de pericardite ativa, endocardite, êmbolo séptico, aborto recente (nas últimas 3 semanas), gravidez e puerpério; o) Infarto do miocárdio recente. 3.3. Manejo da hipertensão arterial: O uso dert-PA para o tratamento do AVC isquêmico agudo implica na neces-
Tabela 2. Manejo da hipertensão arterial prétratamento com rt-PA.

PAD $>110 \mathrm{mmHg}$ ou

PAS $>185 \mathrm{mmHg}$

\begin{tabular}{ll}
\hline METOPROLOL OU & METOPROLOL: (1 amp = $5 \mathrm{ml}, 1 \mathrm{mg} / \mathrm{ml}$ ). \\
LABETALOL** & $\begin{array}{l}\text { Aplicar EV: } 5 \mathrm{mg} \text { a cada } 10 \mathrm{~min}, \\
\text { sendo } 1 \mathrm{mg} / \text { minuto. Máximo: } 20 \mathrm{mg} .\end{array}$ \\
& LABETALOL: $10 \mathrm{mg}$ em 1 a 2 minutos. \\
& Repetir ou dobrar a dose a cada 10 \\
& minutos. Máximo: $160 \mathrm{mg}$. \\
ENALAPRIL** & ENALAPRIL frasco-amp de $1 \mathrm{ml}=1 \mathrm{mg}$, \\
& 5 mg ou 10 mg. Correr $1 \mathrm{mg}$ em no \\
& mínimo 5 minutos a 1 hora. Caso \\
& necessário, 1 a 2 mg pode ser repetido \\
& após 1 hora. Novas doses, porém, \\
& deverão ser dadas num intervalo \\
& mínimo de 6 horas.
\end{tabular}

** Medicamento ainda não disponível no Brasil. *** Em casos de asma, insuficiência cardíaca ou severa anormalidade de condução cardíaca, que contra-indiquem o uso do betabloqueador. PAD: pressão arterial diastólica; PAS: pressão arterial sistólica

sidade de controle rigoroso da hipertensão arterial, pois o risco de hemorragia cerebral correlaciona-se com os níveis pressóricos ${ }^{13,31}$. Durante o tratamento, deve-se estar alerta para o risco de hipotensão medicamentosa. Em pacientes candidatos à terapêutica trombolítica, recomenda-se seguir o protocolo do NINDS rt-PA Stroke Study Group ${ }^{5,13}$, no qual são aceitos os seguintes níveis de pressão arterial: PAD $\leq 110 \mathrm{mmHg}$ e PAS $\leq 185 \mathrm{mmHg}$ (Tabelas 2 e 3).

3.4. Cuidados gerais ${ }^{13,21,31}$ :

3.4.1. Deve haver rigoroso controle neurológico através da NIHSS a cada 6 horas, e sempre que necessário. O aumento do escore de até 4 pontos é sinal de alerta e sugere reavaliação tomográfica.

3.4.2. Não utilizar antitrombóticos (antiagregantes, heparina ou anticoagulante oral) nas próximas 24 horas póstrombolítico.

3.4.3. Não realizar cateterização venosa central ou punção arterial nas primeiras 24 horas.

3.4.4. Não passar sonda vesical até pelo menos 30 minutos do término da infusão do rt-PA.

3.4.5. Não passar sonda nasoenteral nas primeiras 24 horas após a infusão.

3.5. Administração por via EV do rt-PA: Discutir comos familiares ou responsáveis os riscos/benefícios do tratamento e fazer o registro por escrito no prontuário do paciente ${ }^{13}$.

3.5.1. Orientações de uso: O tratamento trombolítico exige controle rigoroso da PA (Tabela 2 e 3). A infusão do rt-PA deve ser realizada segundo alguns preceitos estabelecidos ${ }^{5}$. Após analisar os critérios de inclusão e exclusão, conseguir dois acessos venosos periféricos. Administrar o rt-PA na dose total de 0,9 mg/kg, até um total máximo de $90 \mathrm{mg}$. Injetar $10 \%$ da dose EV em até 1 minuto, eo restante em 60 minu- 
Tabela 3. Manejo da hipertensão arterial durante e após o tratamento com rt-PA.

Monitorizar pressão arterial durante as primeiras 24 horas após iniciar o tratamento.

Medir a cada 15 minutos nas primeiras 2 horas

Medir a cada 30 minutos nas próximas 6 horas

Medir a cada 60 minutos até completar 24 horas

\begin{tabular}{|c|c|c|c|}
\hline & $\mathrm{PAD}>140 \mathrm{mmHg}$ & PAS > 230 & PAS 180 - 230 \\
\hline & & PAD 121- 140 (mmHg) & PAD 105 - 120 (mmHg) \\
\hline METOPROLOL ou ENALAPRIL & & $\begin{array}{l}\text { Administração conforme } \\
\text { a necessidade }\end{array}$ & $\begin{array}{l}\text { Administração conforme } \\
\text { a necessidade }\end{array}$ \\
\hline $\begin{array}{l}\text { NITROPRUSSIATO DE } \\
\text { SÓDIO (lamp = 50mg), } \\
\text { diluída em solução } \\
\text { de glicose } 5 \%\end{array}$ & $\begin{array}{l}\text { 0,5-8 ug/kg/minuto, } \\
\text { fazendo reajustes, } \\
\text { se necessário, a } \\
\text { cada } 10 \text { minutos }\end{array}$ & $\begin{array}{l}0,5-8 \text { ug } / \mathrm{kg} / \text { minuto, fazendo } \\
\text { reajustes, se necessário, a } \\
\text { cada } 10 \text { minutos }\end{array}$ & $\begin{array}{l}\text { 0,5-8 ug/kg/minuto, } \\
\text { fazendo reajustes, } \\
\text { se necessário, a } \\
\text { cada } 10 \text { minutos }\end{array}$ \\
\hline LABETALOL** & & $\begin{array}{l}10 \mathrm{mg} \text { (1a2 minutos) } \\
\text { Repetir ou dobrar a dose a cada } \\
10 \text { minutos até } 160 \mathrm{mg}\end{array}$ & $\begin{array}{l}10 \text { mg(1a2 minutos) } \\
\text { Repetir ou dobrar a } \\
\text { dose a cada } 10 \\
\text { minutos até } 160 \text { mg }\end{array}$ \\
\hline
\end{tabular}

** Medicamento ainda não disponível no Brasil. PAD, pressão arterial diastólica; PAS, pressão arterial sistólica.

tos, se possível com bomba de infusão. Durante a infusão, o paciente deverá estar monitorado em Unidade de AVC ou UT pelo período mínimo de 24 horas para a detecção de quaisquer mudanças no quadro neurológico, sinais vitais, ou evidência de sangramento ${ }^{5,13}$.

4. Sinais de alerta: Determinados fatores aumentam o risco de sangramento no sistema nervoso central. A indicação de rt-PA nestes casos deve constituir decisão altamente individualizada ${ }^{5,13}$ :

4.1. NIHSS $>22$ (associado com maior risco de hemorragia).

4.2. Idade $>80$ anos.

4.3. Abuso de álcool ou de drogas.

5. Complicações hemorrágicas: As complicações hemorrágicas pelo uso de trombolítico frequentemente ocorrem nas primeiras 24 horas de terapia. Ficar alerta para deterioração e do quadro neurológico, náuseas, vômitos, cefaléia e hipertensão aguda. Nesta situação, recomenda-se ${ }^{36}$ :

a. Cessar a infusão frente a qualquer sinal de piora neurológica ou evidência de hemorragia significativa.

b. Certificar-se de que duas veias periféricas estejam sendo infundidas com cristalóides.

c. Submeter o pacientea TC de crânio para a confirmação do diagnóstico de sangramento.

d. Solicitar os seguintes exames laboratoriais: hematócrito, tempo de protrombina, TTPa e fibrinogênio. e. Infundir preferencialmente 6 a 8 unidades de crioprecipitado ou 2-3 unidades de plasma fresco. Se houver continuidade da deterioração clínica após 4-6 horas, utilizar hemoderivados de acordo com o coagulograma. Repetir a infusão de crioprecipitado se fibrinogênio baixo, ou administrar o plasma fresco se existir alteração de TP ou TTPa. Infundir 6 a 8 unidades de plaquetas se estiverem em nível baixo ou se o paciente estava em uso de drogas antiplaquetárias.

f. Infundir concentrado de glóbulos suficiente para manter o hematócrito adequado.

g. Infundir fluidos para tratar a hipotensão.

h. Nos casos de hemorragia no sistema nervoso central, considerar uma consulta neurocirúrgica e hematológica.

\section{COMENTÁRIOS}

1. Recomenda-se o registro em prontuário da discussão sobre os riscos e benefícios com os familiares e/ou paciente ${ }^{13}$.

2. Há controvérsia quanto ao uso de administração do rt-PA endovenoso entre 3-6 horas 5,37,38.

3. Pode haver benefício no uso intra-arterial de trombolíticos da artéria cerebral média até 6 horas usando próuroquinase (nível de evidência I grau B) ${ }^{39}$.

4. A oclusão da artéria basilar pode ser tratada em centros de referência por via intra-arterial após 3 horas (nível de evidência IV grau C) ${ }^{40,41}$.

5. Não há estudos prospectivos que justifiquem o uso de rtPA em pacientes menores que 18 anos. 


\section{REFERÊNCIAS}

1. Lessa I. Epidemiologia das doenças cerebrovasculares no Brasil. Rev Soc Cardiol Estado de São Paulo 1999;4:509-518.

2. Biller J, Love BB. Ischemic cerebrovascular disease. In Bradley WG, Daroff RB, Fenichel GM, Marsden CD (eds). Neurology in Clinical practice. 3.Ed. Boston: Butterworth-Heinemann, 2000:1125-1166.

3. Bonita R. Epidemiology of stroke. Lancet 1992;339:342-344.

4. Wolf PA, Kannel WB, D'A gostino RB. Epidemiology of stroke In Ginsberg MD, Bogousslavsky J (eds). Cerebrovascular disease: pathophysiology, diagnosis, and management. Boston: Blackwell Science, 1998:834-849.

5. The National Institute of Neurological Disorders and Stroke (NINDS) rt-PA Stroke Study Group. Tissue plasminogen activator for acute ischemic stroke. N Engl J Med 1995;333:1581-1587.

6. The NINDSt-PA StrokeStudy Group. Generalized efficacy of t-PA for acute stroke: subgroup analysis of the NINDS t-PA stroke trial. Stroke 1997;28:2119-2125.

7. KasteM, Olsen TS, OrgogozoJM, et al. Organization of strokecare: educa tion, strokeunits and rehabilitation. Cerebrovasc Dis 2000;10(Suppl 3):1-11.

8. Fisher M, Garcia JH. Evolving stroke and the ischemic penumbra. Neurology 1996;47:884-888.

9. Fisher M. Anti-ischemic stroketherapy. In Ginsberg MD, Bogousslavsky J (eds) Cerebrovascular disease: pathophysiology, diagnosis, and management. Boston: Blackwell Science, 1998:1878-1886.

10. Hacke W, Kaste M, Fieschi, et al. for the European Cooperative Acute Stroke Study (ECASS). Intravenous thrombolysis with recombinant tissue plasminogen activator for acute hemispheric stroke. JAMA 1995;274:1017-1025.

11. Caplan LR. Treatment. In Caplan LR (ed). Stroke: a clinical approach. Boston: Butterworth-Heinemann, 2000:115-161.

12. Adams HP, BrottTG, Furlan AJ, etal. Guidelines for thrombolytictherapy for acute stroke: a supplement to the guidelines for the management of patients with acute ischemic stroke. Circulation 1996;94:1167-1174.

13. HackeW, KasteM, Olsen TS, et al.. A cutetreatment of ischemic stroke. Cerebrovasc Dis 2000;10(Suppl 3):22-33.

14. European Stroke Initiative (EUSI). Recommendations for stroke management. Cerebrovasc Dis 2000;10(Suppl 3):1-34.

15. A boderin I, Venables G. Stroke management in Europe: Pan European Consensus Meeting on StrokeManagement. J Intern Med 1996;240:173-180.

16. Quality Standars Subcommitee of the American Academy of Neurology. Practice advisory: thrombolytic therapy for acuteischemic stroke. Neurology 1996;47:835-839.

17. Adams H, BrottT, Crowell R, et al. Guidelines for management of patients with acute ischemic stroke: a statement for health care professionals from a special writing group of the Stroke Council, American Heart Association. Stroke 1994;25:1901-1914.

18. Gorelick P, Sacco R, Smith D, et al. Prevetion of first stroke: a review of guidelines and a multidisciplinary consensus statement from the $\mathrm{Na}$ tional Stroke Association. JAMA 1999;281:1112-1120.

19. WHO Task Force on stroke and other cerebrovascular disorders. Recommendations on stroke prevention, diagnosis, and therapy. Stroke 1989;20:1407-1431.

20. Kaste $M$, Thomassen $L$, Grond $M$, et al. Thrombolysis for acute stroke: a consensus of the 3rd Karolinska Stroke Update, October 30-31, 2000. Stroke 2001;32:2717-2718.
21. SociedadeBrasileira deDoenças Cerebrovasculares. Primeiro consenso brasileiro do tratamento da fase aguda do acidente vascular cerebral. Arq Neuropsiquiatr 2001;59:972-980.

22. Culebras A, KaseCS, Masdeu JC, et al. Practice Guidelines for the use of imaging in transient ischemic attacks and acute stroke: a report of the Stroke Council, American Heart Association. Stroke 1997;28:1480-1497.

23. Ferro JM. Conduta inicial no paciente com acidente vascular cerebral isquêmico agudo. Rev Soc Cardiol Estado de São Paulo 1999;4:570-577.

24. Fisher M, Prichard JW, Warach S. New magnetic resonancetechniques for acute ischemic stroke. JAMA 1995;274:908-911.

25. Albers GW. Diffusion weighted MRI for evaluation of acute stroke. Neurology 1998;51(Suppl 3):47-49.

26. Fisher M,Albers GW. A pplications of diffusion perfusion magneticresonance imaging in acute ischemic stroke. Neurology 1999;52:1750-1756.

27. Kidwell CS, Saver JL, Mattiello J, et al. Thrombolytic reversal of acute human cerebral ischemic injury shown by diffusion/ perfusion magnetic resonance imaging. Ann Neurol 2000;47:462-469.

28. Teasedale G, Jennett B: Assesment of coma and impaired consciousness. A practical scale. Lancet 1974;2:81-83.

29. Brott $T, A$ dams HP, Olinger $C P$, et al. Measurements of acute cerebral infarction: a clinical examination scale. Stroke 1989;20:864-870.

30. Lyden P, Brott T, Tilley B, et al. I mproved reliability of the NIH Stroke Scale using video training. Stroke 1994;25:2220-2226.

31. Lewandowski C, Barsan W. Treatment of acute ischemic stroke. Ann Emerg Med 2001;37:202-216.

32. Donnan GA, Davis SM, Chambers BR, et al. for the Australian Streptokinase(ASK) Trial Study Group. Streptokinasefor acuteischemic stroke with relationship to time of administration. JAMA 1996;276:961-966.

33. Multicenter Acute Stroke Trial European Study Group. Thrombolytic therapy with streptokinase in acute ischemic stroke. N Engl J Med 1996;335:145-150.

34. Multicenter Acute Stroke Trial - Italy (MAST-I) Group. Randomized controlled trial of streptokinase, aspirin, and combination of both in treatment of acute ischemic stroke. Lancet 1995;346:1509-1514.

35. Yasaka M, O'Keefe GJ, Davis SM, et al. for the Australian Streptokinase Trial Study Group. Streptokinase in acute stroke: effect on reperfusion and recanalization. Neurology 1998;50:626-632.

36. The NINDS rt-PA StrokeStudy Group. Intracerebral hemorrhage after intravenoust-PA therapy for ischemic stroke. Stroke 1997;28:2109-2118.

37. HackeW, Kaste M, Fieschi C, et al. Randomized double-blind placebocontrolled trial of thrombolytic therapy with intravenous alteplase in acute ischaemic stroke (ECASS II). Lancet 1998;352:1245-1251.

38. Wardlaw J, Warlow C. Thrombolytic therapy for acuteischaemic stroke: the updated Cochrane database of systemic reviews metaanalysis. Cerebrovasc Dis 1999;9:124.

39. Ferland AJ, Higashida R, Wechsler L, et al. Intra-arterial prourokinase for acute ischemic stroke: the PROACT II Study. A randomized controlled trial. JAMA 1999;282:2003-2011.

40. Egan R, Clark W, Lutsep H, et al. Efficacy of intra-arterial thrombolysis of basilar artery stroke. J Stroke Cerebrovasc Dis 1999;8:22-27.

41. HackeW, Zeumer H, FerbertA, et al. Intra-arterial thrombolytic therapy improves outcome in patients with acute vertebrobasilar occlusive disease. Stroke 1988;19:1216-1222. 